\title{
Cultivating Invisible Impact with Deep Technology and Creative Destruction
}

\author{
Joshua E. Siegel \\ jsiegel@msu.edu | Department of Computer Science and Engineering, Michigan State University, 428 \\ South Shaw Lane, Room 3115, East Lansing, Michigan, 48824 United States \\ Sriram Krishnan \\ sriram@alum.mit.edu | Research Affiliate, Department of Mechanical Engineering, Massachusetts \\ Institute of Technology, Cambridge, Massachusetts, United States

\section{Letter from Academia}

\begin{abstract}
The term "Deep Tech" is prevalent in industry but ill-defined. In this extended letter, we define Deep Tech (technology that is difficult to develop today, with the potential to become a pervasive and easy-to-implement basic need in the future) and consider its role in evolving businesses. We suggest how organizations can position themselves to take advantage of a coming wave of innovation, with emphasis placed on Creative Destruction's role (cannibalizing product lines) in creating sustaining enterprises. Understanding the diffusion of technical innovation from hard-to-implement exciter to commodity basic need will encourage leaders to rethink their current offerings to play in a future-thinking space setting these companies apart from the competition. We provide examples drawn from successful organizations.
\end{abstract}

Keywords. Deep Technology; Deep Tech; Creative Destruction; Kano Model; Technology Diffusion.

Cite paper as: Siegel, J.E., Krishnan, S., (2020). Cultivating Invisible Impact with Deep Technology and Creative Destruction - Letter from Academia, Journal of Innovation Management, www.open-jim.org, 8(3), 619. 


\section{Emerging Technologies Can Become Essential Needs}

Today's mundane technology was recently bleeding edge. Consider air conditioning, an engineering masterpiece once only for elites and today, so pervasive that its absence is noteworthy.

Favoring conspicuous successes, businesses often overlook the beauty in "boring." Rather than struggling to visibly pioneer, organizations may be better-served by cultivating covert disruption. This invisible innovation requires commoditizing complex and recently feasible "Deep Technologies," technologies that demand significant intellectual and economic capital to pursue, and with the potential to become seamless, scalable and transparent solutions.

Innovations bring about transformation not upon their introduction, but rather through democratization. It is laudable for an organization's offerings to be taken for granted, and companies making previously deep technologies indispensable reap social and economic rewards.

The primary goal of this letter is to make the term Deep Technology (Deep Tech) accessible and approachable not only to startups, but also to individuals and large-scale enterprises. We present a nuanced definition of Deep Tech, consider how Creative Destruction's cannibalization of strongperforming products and services supports its adoption by enabling long-term welfare increases, and explore technology's distillation and diffusion from bleeding-edge to basic-need.

A secondary goal is for readers to understand, embrace, and be able to operationalize Deep Tech within their industries, and we do so by exploring the nature of Deep Tech through a snapshot of ongoing activities as well as the means through which practitioners may learn and implement their own technical transformations. A tertiary goal unpacks examples of contemporary Deep Tech transformations to provide representative visions of a transformed future, leading into discussion about how leaders across verticals may synchronize efforts to build a brighter technology future.

We exemplify how organizations have and will continue to evolve to capture value from IoT's growing pervasiveness and seamlessness, and close by discussing how the changes and challenges helping and hindering IoT's growth generalize across industries. We specifically focus on leadership's role in cultivating an environment supportive of Deep Technologies, Creative Destruction, and capitalizing upon becoming the status-quo.

\section{Deep Technology Is Hard... At First}

You may have seen it online or in print: Deep Technology, technology to save - or change - the world. Or was it technology that costs a lot and takes time to develop? Or wait, was it instead technology requiring skill to implement and a grander, socially-minded vision for the future? No, that can't be it, it was technology disrupting traditional industries.

Deep Technology appears to have been coined as a term of art in 2014 by Swati Chaturvedi, CEO of Propel(x). (Chaturvedi, 2014) At the time, she defined Deep Technology startups as "companies founded on a scientific discovery or true technological innovation." This definition purposefully allows for interpretation, as future technologies may not be comprehensible or describable with today's thinking and language. As a result of the definition's flexibility, the term 
has since been co-opted as a means of justifying significant time, economic, and intellectual capital expenditure in cultivating new businesses and business lines.

Deep Tech has become a catch-all for futurology-cum-high tech used by individuals, startups, venture funds, and consultancies. Today, it may refer to hard or soft sciences, big or small problems with or without social impact, and radical or sustaining innovation. As with the Internet of Things before it, and Digital Transformation in parallel, businesses, funds, and entrepreneurs use Deep Tech as a "flavor enhancer" - MSG for their offerings, designed to captivate and engage. When pressed, few can define Deep Tech, and fewer still agree upon all but its basest of elements. Playing fast-and-loose with the term erodes its differentiating power and risks diminishing the value of something potentially industry-defining. If organizations can't agree on what, exactly, Deep Tech is, then how can they plan to implement it properly?

In this section, we refine and sharpen Ms. Chaturvedi's definition in order to recapture the essence of what Deep Tech is, what it does, and what it might become - so that practitioners might better understand, embrace and develop its capabilities. To this end, we first present a comprehensive and extensible definition of Deep Tech encapsulating the essence of the concept. Our experience as co-authors informs this definition: one of us founded the Deep Tech Lab at Michigan State University and runs the Deep Tech Bootcamp at MIT, the other is an industry practitioner and researcher versed in transformative technology (exponential growth, in the case of solar) and industry evolution. Our definition is as follows:

A "Deep" Technology was impossible yesterday, is barely feasible today, and will quickly become so pervasive and impactful that it is difficult to remember life without. Deep Tech solutions are reimaginations of fundamental capabilities that are faithful to real and significant problems or opportunities, rather than to one discipline.

Deep Tech's fundamental capabilities comprise sensing, connectivity, computation, inference, actuation, and control. Sensing allows systems to capture information about themselves and their context, connectivity moves information to the location of highest-utility, and computation and inference algorithmically distill information into insight. Actuators affect a system's state, while inference-informed control precisely modulates outputs to create a responsive feedback loop to maximize impact.

Supporting these capabilities are advances in low-cost, low-power and pervasive sensing, ubiquitous connectivity, and efficient and performant parallelized computing. Improvements in algorithm design, standardized software, and network architectures enhance system scalability, security, and performance to better meet evolving needs. Contemporary, Deep Tech-supporting innovations include distributed elastic computing, additive manufacturing, gene-editing, voice services, microsatellites, wireless power and improved battery chemistries. By the time you read this, these forward-looking concepts may already be passé, as today's Deep Tech diffuses into High Tech, Tech, and eventually, basic need.

Across domains, Deep Tech capabilities and un-siloed thinking help innovators address both green- and brown-field opportunities. For either, businesses may need to disrupt ongoing successes to create more significant long-term and sustaining impact. 


\section{Deep Tech's March Towards the Mainstream}

While it is difficult to quantify the past, present, and future state of Deep Tech as we define it, we find that, at least with Deep Tech's expanded definition, its market size is substantial, growing, and global, with pent-up excitement for its adoption and entrepreneurship. Surveying the state of Deep Tech under a definition similar in scope to Ms. Chaturvedi's, BCG reports category investments as growing $22 \%$ annually from 2015 to 2018 , reaching nearly $\$ 18$ B (Portincaso \& de la Tour, 2019). Deep Tech companies have more private funding events than their shallower technology counterparts, this largely championed by venture capital; the U.S. and China lead with $81 \%$ of global private investments in Deep Tech from 2015-18, and 53\% of Deep Tech companies U.S.-based (Portincaso \& de la Tour, 2019), though Europe's own Deep Tech Renaissance was itself on track for $\$ 3.5 \mathrm{~B}$ of investment across 600 deals in 2017 (Dealroom.co, 2017). In the years since, funding has only grown, with $€ 7.7$ invested in 2019 (Fresneau, 2020).

Some Deep Tech companies may be familiar to JIM readers, these playing in both traditional and emergent verticals. For example, in recent years, Deep Tech turned car rental on its head. It was previously a daunting affair: call ahead to a large company, wait on hold to make a reservation, share your personal details with an unsavory character, stand in line, wait for the attendant to get your keys and scan your license... and then find out they don't have the car you reserved. ZipCar was founded to solve this problem, spawning the first Deep Tech car rental agency - its core innovation being to put the vehicle's key (indirectly) on the Internet, removing both costly parking lots and time-consuming human interaction from the rental equation (Bernardi, Sarma, \& Traub, 2017). In doing so, it launched the Mobility on Demand revolution.

In the two decades since its inception, Zipcar has evolved to become a default option for urban consumers and sub-day rentals. Yet, limited vehicle options may create friction. Presently, Turo is another company using Deep Tech to make car renting painless: it uses the Internet of Things, mobile devices, and online marketplaces to remove big business from the rental process. Anyone can put their own, privately-owned vehicle online and set their rental rules and rates - with driver details, from phone number to insurance information, all shared automatically. Soon, the Deep Tech-driven evolution of Mobility on Demand may have a market totaling more than $\$ 250 \mathrm{~B}$ (Bhutani \& Saha, 2019).

Peloton is another example of a high-profile Deep Tech business. Traditional gyms suffer from high capital costs and poor utilization, while trainers struggle to fill or keep up with large class sizes. Peloton uses Deep Tech - the Internet of Things, smart sensors, and advanced analytics - to put interactive fitness classes on the Internet. Users, rather than gyms, pay for (and house) equipment and gain access semi-individualized instructor attention. The user eliminates a commute and gains the ability to work out cost-effectively in private, while Peloton's "virtual gym" unbundles the space and equipment requirements to shift the infrastructure capital costs and divide instructor compensation among a large, geographically-distributed userbase. The resulting experience is similar to that of a one-on-one class, offering choices of a particular playlist or workout style with occasional metric-driven words of encouragement - and the added ability to opt-into sharing data, participating in gamified events, or making social the gymgoing experience at the push of a button. And instructors, rather than risking their wages to a small-scale, local audience, leverage their reach to live as professional athletes (Chen, 2019). 
Peloton, as with ZipCar, launched its own revolution. Its September 2019 IPO valued it at $\$ 7.2 \mathrm{~B}$ (Hirsch \& Lucas, 2019) and its unique offering has thrived under COVID-19 related stay-at-home restrictions, with its valuation increasing more than three times from January to September 2020 (Yahoo!, 2020). The global digital fitness market for which Peloton is a trendsetter is expected to grow at a more than $30 \%$ compounded rate for the next three to five years (Lockwood, Bowmer, \& Jones-Pritchard, 2020).

In agriculture, Deep Tech plays a similar role in market-shaping and growing. Engineered seeds are perhaps the most-visible example. This technology is nascent, leveraging recent advances in gene editing based upon data-driven engineering to create seeds that are robust across environmental conditions, resistant to chemical agents, and with improved yield and quality. Soon, this market may be worth $\$ 30 \mathrm{~B}$ (Fortune Business Insights, 2019) and the concept has already been the basis of numerous high-profile lawsuits (Eliane Peschard, 2019). These are just a few examples of how Deep Tech has revolutionized industries. Later in this letter, we explore the Deep Tech exemplars of IoT and agriculture transformation ecosystem in depth.

While Deep Tech may transform businesses and their operations to the core, not all benefits of Deep Tech are economic. Deep Tech startups may seek to solve intractable societal challenges by creating new capabilities and sprouting new businesses in as yet untapped global markets. In a survey of 8,600 global Deep Tech companies, more than half aimed to tackle at least one of the United Nations' Sustainable Development Goals (Portincaso, de la Tour, \& Soussan, 2019).

But it's not just "random acts of Deep Tech." Rather, there is an emergent focusing of what was previously unconstrained innovation. As the Deep Tech moniker has spread, so too has the ecosystem for learning and implementing Deep Tech grown. There are today accelerators for Deep Tech startups such as DeepTech4Good (Deep Tech 4 Good, 2018) and training Bootcamps focusing on blending technology and entrepreneurial innovation, such as the author's own (MIT Bootcamps, 2020). These focus less on specific technologies, and more on modes of thinking, including convergent innovation and innovation mindsets, and new business or interaction models. Accelerators and training programs emphasize building long-term and sustaining visions predicated upon technology that today only might be possible, while all the while reminding - or informing - participants that Deep Tech used to be cost-prohibitive and complex-to-build, whereas modern tools reduce cost, complexity, and knowledge barriers to development and deployment in domains such as software platforms, applications, and analytics. Truly, now is the best time to be working with technologies that do not yet exist.

\section{Creative Destruction Lucratively Loses Money}

On the way towards pervasive adoption, Deep Tech innovations may disrupt incumbent solutions. "Creative Destruction" is necessary to ensure that legacy implementations are fully replaced with the best-available technology. Consider how automobiles replaced horses - profitable carriage industries were forced to reskill or shutter. Yet, other industries such as wheelbuilding experienced a boom, and society is now arguably better off.

Creative Destruction is a necessary step toward embracing technological improvements, and organizational thinking must shift from traditional defensive strategies focused on protecting 
established offerings towards leveraging bleeding-edge innovation and opportunity-focused convergent design to best pioneer transformative Deep Technologies.

Recent advances have made Creative Destruction increasingly viable by easing technology prototyping and experimentation and reducing cost, complexity, and time to market. Today, managers with little technical experience can leverage assistive tools to become the front-line in implementing emerging technologies. However, the availability of tools alone does not create immediate paths to Deep Tech viability.

Organizations must embrace Creative Destruction by celebrating cross-functional, cross-domain knowledge, and new (*aaS - blank-as a service, where blank includes Platforms, Software, Infrastructure, and Desktops, among other concepts) and evolving business models (multi-stakeholder relationships, capitalizing latent value in data, and shifting from single-sale to ongoing customer relationships). Successful companies understand the value hidden at the intersection of existing technologies, capabilities, products, and services, and are willing to work with players across verticals and horizontals to unlock these interstitial value creation opportunities.

\section{Transformative Technologies Become Transparent}

Regardless of whether innovation involves Creative Destruction or stems from another process, companies should consider the value in making their offerings intentionally mundane. Many transformative innovations, whether radical or sustaining, begin as visible and exciting disruptors, and over time, become transparent basic needs following a process known as the Kano Model.

The Kano Model (Kano, Seraku, Takahasi, \& Tsuji, 1984) draws the arc from how technologies exceeding expectations (delighters) quickly become basic needs (must-be qualities) as adoption and familiarity increases. With air conditioning, the technology was initially a delighter. Now it is pervasive, notable only in its absence. The same happened with mobile phones -- technology's transition toward basic need is a virtuous cycle that encourages the technology's use and further development, lowering costs and increasing access, or eroding margin and heightening volume.

While many successful organizations seek conspicuous innovation, played skillfully, democratization of Deep Technology can outstrip the value created through flashier, less-pervasive solutions. Organizations should increasingly seek to make this "invisible advantage" an incipient part of a solution's roadmap.

The interplay among the Kano Model, Hype Cycle, Adoption Cycle, and our definitions for Deep Tech, High Tech, and Tech are shown in Fig. 1.

\section{Innovation and Creative Destruction in Robotic Automation}

Agriculture has long been an indicator of civilization. Until recently, land was tilled by manually operated equipment, limiting humans' ability to manage acreage.

Recent advances have allowed farming equipment to be automated, reducing costs, improving 


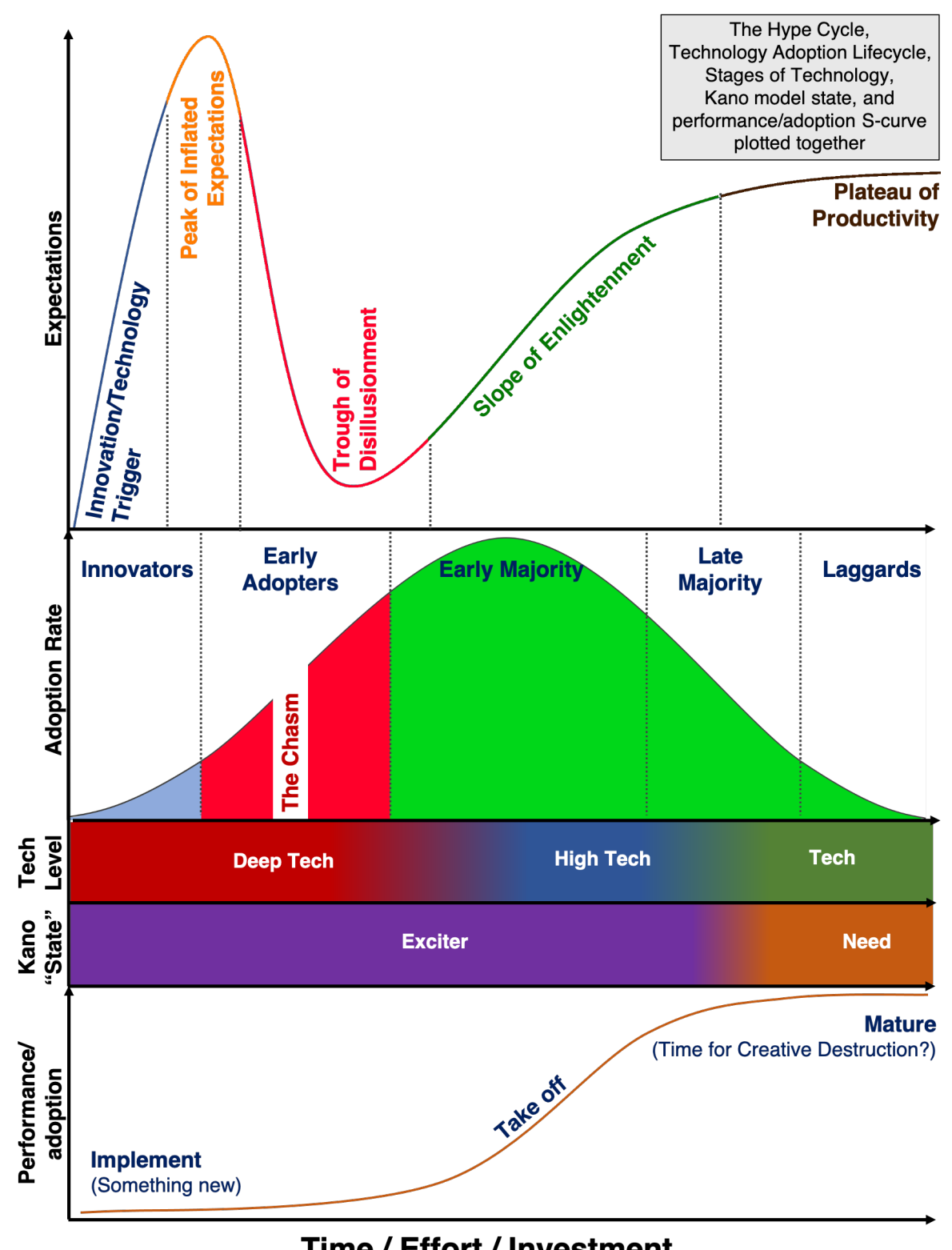

Time / Effort / Investment

Fig. 1. Gartner's Hype Cycle (top) shows one view of "typical" technology evolution, from innovation trigger, through implementation challenges and discovery, to widespread applicability. The Technology Adoption Lifecycle (second from top) shows the adoption rate for technology throughout its development; innovators and early adopters put up with imperfection and challenges while the majority and laggards require technology to be ironed-out. The Stages of Technology and Kano State (third from bottom and second from bottom) show how Deep Tech becomes High Tech (technology non-practitioners deem to be cutting-edge) and then simply Technology, while at the same time moving from exciter to basic need. The performance/adoption S-curve shows a performance curve as technology advances and how maturity is the optimal time for Creative Destruction. 
yield, and supporting field expansion. This confluence of enabling capabilities -- sensing, connectivity, inference, and action -- met a real, timely, and significant need.

Robotic Automation is an example of democratized Deep Technology. Ten years ago, the requisite capabilities were nascent. Five years ago, the technology was costly and imperfect. Today, the technology is more affordable, works well, and is invisible to consumers (except through higher quality produce and lower prices). Tomorrow, it may be invisible to farmers ("what do you mean, I have to drive this tractor?").

Getting to this point required Creative Destruction. As an example, John Deere used to sell tractors, but now it is in the business of Farming as a Service. By offering connected services, integrating software into the repair process, and creating future opportunities for software updates and feature unlocking, it changed from a vendor focused on single point of sale to one with potential for annuity revenues and increased customer touchpoints. John Deere cannibalized its existing business to remain a leading agricultural equipment player.

Though this innovation was first seen as radical and drew criticism, the choice to Creatively Destruct existing business lines helped rapidly democratize agricultural equipment software solutions, and in turn, high automation. In a few short years, John Deere and others have accelerated Robotic Automation's progression through the Kano Model, taking it from an exciting (costly) feature to basic (affordable) need and creating a new standard for "must have" features. Increased Deep Tech pervasiveness has served the company well, growing its revenues and valuation.

\section{Watching The Internet of Things Grow Up}

While Robotic Automation is far along the Kano Model, another compelling example of Deep Tech is a technology partway between exciting capability and basic need: the Internet of Things. Until a few years ago, its enabling technologies were impossible, or at least, cost-prohibitive.

We saw how not long ago, we had to rent cars at bespoke brick and mortar locations. With RFID keycard access and cellular connectivity, IoT led to businesses such as ZipCar, which granted consumers broader access to rental cars by putting the key, and their driver's license, on the Internet. At the same time, IoT granted rental agencies access to more locations than ever.

These technologies for connectivity, location tracking, secure digital contracts and remote access management matured individually, but as market opportunities crystalized for IoT, they began to evolve in aggregate. Because the technologies coevolved, they became easier to combine - and today, there are markets where individual owners can rent their under-utilized cars to strangers at an arm's length, or where anyone with a cellphone can rent an electric scooter by the minute.

With the range of possibilities limited only by imagination, problems and opportunities like vendor lock, cybersecurity, energy efficiency, and interoperability emerged. Organizations saw the need to address these problems and began to work to solve them.

Today, we are part way towards IoT's maturation into a fundamental human need. Location 
services are pervasive for targeted advertising. Wearables invisibly track our movement. Vehicles learn from telemetry data. Smart homes proactively optimize our comfort. These services rely on Deep Tech's fundamental capabilities of sensing, connectivity, computation, inference, actuation, and control to create a more responsive and engaging world.

Getting to this point has not been easy. Organizations have had to not only invest in Deep Technologies, but also changed in structure and approach. They must continue to do so to complete IoT's enablement and to tap into the resulting value capture. There is much to be learned about democratizing Deep Tech from studying IoT's ongoing rollout.

\section{What Can We Learn from IoT?}

IoT companies actively employ Creative Destruction, in spite of ongoing work to address the teething pain of its rollout. While many companies initially forced vendor-lock by limiting interoperability or requiring the use of a proprietary standard, major players aim to develop common architectures capable of enabling the seamless interaction of portfolio of technologies and applications - destroying contemporary, revenue-generating solutions in the hopes of unlocking greater potential. The shift to an IoT-enabled future will require a deeper understanding of the customer experience, with empathy helping to build new IoT-enabled value-added services that improve comfort, convenience, efficiency, performance, and safety.

As IoT becomes increasingly transparent, its remaining friction points become visible. While user convenience improves with the personalization and localization services afforded by IoT, and while organizations stand to benefit from improved safety, efficiency, and the ability to access new markets enabled by IoT's richer data generation and control capabilities, practitioners must address interoperability and security and privacy concerns responsibly to facilitate the technology's most successful implementation.

Related conversations highlight the need for IoT's governance, and ways to facilitate publicprivate partnerships at all levels, for the purpose of shared value creation and preparing organizations and their workforce for an IoT-enabled future. In this manner, IoT's rollout presents a compelling example that business leaders may want to emulate. But, how can these and other lessons learned from IoT inform organizations on how best to cultivate Deep Tech?

\section{How can Organizations Embrace Deep Tech and Creative Des- truction?}

Technologies neither exist nor are developed in vacuums. Implementing Deep Tech requires leaders and organizations to shift their mindset to "know what they don't know," and to adopt a "proudly found elsewhere" mentality to uniquely consolidate solutions drawn from disparate entities, rather than the traditional "winner-takes-all" thinking.

There is an emerging consensus that shared value creation through public-private partnerships and ecosystem building efforts are needed in implementing Deep Technologies (Siegel, et al., 2019). By establishing public-private partnerships, companies may reduce the risk of access to capital and utilize public infrastructure as an opportunity for a scale-up roadmap. Exam- 
ples of such partnerships are seen around the world in smart-cities with connected traffic solutions, (Ericsson, 2018) IoT-connected agricultural farms, vineyards or mangroves (Modawal, 2019).

By curating startup ecosystems, established companies may partner with and help cultivate a cohort of Deep Tech startups. Large companies see Deep Tech startups as new sources of innovation and for reducing the risk of demand and development bets (products or concepts in search of a market or yet-to-be-proven) (Soussan \& de la Tour, 2017). Deep Tech startups benefit from larger organizations' access to seed funding, market context and available test-beds for pilots. Absorbing such innovation within large companies requires two-speed organizations (the larger, slower incumbent and the nimble, innovative startup) that need to get synchronized eventually. Our earlier discussion on the shift in mindset required for Deep Tech and its evolution will serve well both entrepreneurs and business leaders as they envision partnerships. It is not only big-helping-little, and little-helping-big. Peer organizations glean from one another heardlearned lessons in technology or business development, and realize the fruits of each other's' investments into technology research laboratories and talent cultivation.

These partnerships solve problems not by using the thinking employed in creating them, but rather by addressing multiple stakeholders' latent needs with emerging capabilities. More than traditional industries, and perhaps counterintuitively, Deep Tech requires a market-driven approach to innovation rather than a technology-driven approach. The green fields are so broad that having a specific problem helps to focus innovation. To best address identified opportunities, innovators must frame solutions in a descriptive, rather than prescriptive, manner, to avoid early lock-in down a potentially-suboptimal course.

Embracing Deep Tech requires a culture of leadership, celebrating learning, experimentation, and innovation that both nurtures and creates industries. The formalization of roadmaps capable of generating value from the present continuously into the future is imperative, and embracing sustaining, widely adopted, lower-margin technical solutions is every bit as important as high-margin, low-volume whiz-bang marketable tech. The organizations that are best poised to succeed will have infrastructure in place to support and rapidly scale both traditional and nontraditional IT and OT implementations. Importantly, practitioners should note that it is no longer a threat for a company to innovate itself into oblivion by creating a sustaining innovation that makes future sales impossible.

The Deep Tech innovation cycle will increasingly become continuous, rather than cyclical, requiring managers to help identify, develop, and deploy solutions both directly and indirectly including by helping to clear technical and organizational hurdles. Managers should similarly identify and build a supportive scaffold for new ideas of sponsors, champions, integrators, and customers, empowering every participant to play an active role in designing new solutions.

For talent management, Deep Technologies will benefit from hiring cross-discipline thinkers skilled in both theory and practice (or participating in "bootcamps" to train existing employees), flat organizational hierarchies, and a willingness to find a nascent idea and to cultivate it fully rather than moving quickly onward for the "next big win." Leaders must be able to shape their teams to map out complex, multi-stakeholder interactions, and have a mind for contingency identification 
and planning. Knowing when (not) to implement Deep Technology, and the ability to discern opportunity from hype, will differentiate successful teams.

\section{A Call to Action}

Deep Technology has the potential to reinvent businesses for the better, maximizing impact, scale, profit, and social welfare in concert. To unlock these benefits, leaders must pay attention to both market trends and the fast-changing world of technical innovations, championing Deep Tech within their organizations and embracing both internal and external insights. At times, this may involve disrupting successful solutions using Creative Destruction in order to realize something with greater lifetime value.

The organizations that find a timely pairing between a real and significant opportunity and a fast-developing technology or set of technologies, and a willingness to rapidly shift their organizational alignment to match this direction, have the potential to realize huge success. Having cross-disciplinarily trained people, and a corporate culture embracing radical innovation with a focus on its democratization - even if the technology is "unsexy" - helps to support this transformation.

We encourage readers to assess their organization's current state and identify opportunities to become tech-progressive, and to reflect on whether business as usual could turn into broader impact through Deep Tech disruption.

This letter aimed to make Deep Technology accessible and approachable through offering a clearer-cut definition of the term and explaining how businesses might - and already are - implementing Deep Tech. We also set out to improve readers' understanding of how Deep Tech might be operationalized across industries, by way of example and exploration of the concept's relationship to traditional business development processes, and to unpack examples of contemporary Deep Tech Transformations. In our exploration of the term's history and emerging significance, and through the examples provided, we believe this letter addresses our aims, and that readers should depart having a better understanding of and willingness to embrace Deep Technology in their own lives and organizations. We hope in the future to continue the conversation, and endeavor to compile a generalizable framework for Deep Tech Innovation across industries and scales.

\section{Acknowledgements}

There was no financial support received in conducting this research or in writing this manuscript.

\section{References}

Bernardi, L., Sarma, S. E., \& Traub, K. (2017). The inversion factor: How to thrive in the IoT economy. Cambridge: MIT Press.

Bhutani, A., \& Saha, P. (2019, November 7). Global Mobility on Demand Market worth $\$ 250 \mathrm{bn}$ 
by 2026. Retrieved from Global Market Insights: https://www.gminsights.com/pressrelease/ mobility-on-demand-mod-market

Chaturvedi, S. (2014). Retrieved September 10, 2020, from Propel(x): https://blog.propelx.com/ what-are-deep-technology-startups-and-why-are-they-good-investments/

Chen, T. P. (2019, May 7). Peloton Instructor: 'We Are Treated Like Professional Athletes'. The Wall Street Journal.

Dealroom.co. (2017). European deep tech continues to attract large investment. Retrieved from State of European Tech: https://2017.stateofeuropeantech.com/chapter/deep-tech/article/ european-deep-tech-seize-global-opportunities/

Deep Tech 4 Good. (2018, November 4). Project: Helping Deep Tech startups accelerate their development. Retrieved from Deep Tech 4 Good: https://www.deeptechforgood.eu/theproject/

Eliane Peschard, K. (2019, October 31). Monsanto wins $\$ 7.7 \mathrm{~b}$ lawsuit in Brazil - but farmers' fight to stop its 'amoral' royalty system will continue. Retrieved from The Conversation: https://theconversation.com/monsanto-wins-7-7b-lawsuit-in-brazil-but-farmers-fight-tostop-its-amoral-royalty-system-will-continue-125471

Ericsson. (2018). World's first Nationwide, real-time traffic service solution; provided by Government. Erisson. Retrieved from Ericsson.com: https://www.ericsson.com/en/cases/2017/smarttalking-traffic-ecosystem

Fortune Business Insights. (2019, May). Genetically Modified Seeds Market Size, Share and Industry Analysis By Crop (Corn, Soybean, Cotton, Canola and Others), and Regional Forecast 2019-2026. Retrieved from Fortune Business Insights: https://www.fortunebusinessinsights.com/ industry-reports/genetically-modified-seeds-market-100389

Fresneau, V. (2020, May 14). 10 promising European deeptech startups leading us into the future. Retrieved from EU Startups: https://www.eu-startups.com/2020/05/10-promising-deeptech-startups/

Hirsch, L., \& Lucas, A. (2019, September 26). CNBC. Retrieved from Peloton slides after opening below IPO price in market debut: https://www.cnbc.com/2019/09/26/peloton-pton-ipo-stockstarts-trading-at-27-per-share.html

Kano, N., Seraku, N., Takahasi, F., \& Tsuji, T. (1984). Attractive Quality and Must-be Quality. Journal of the Japanese Society for Quality Control, 39-48.

Lockwood, B., Bowmer, W., \& Jones-Pritchard, M. (2020, April). Trends in Digital Fitness. Retrieved from Lincoln International: https://www.lincolninternational.com/perspectives/trendsin-digital-fitness/

MIT Bootcamps. (2020). MIT Deep Technology Bootcamp. Retrieved from MIT Bootcamps: https://learn-bootcamp.mit.edu/deep-technology

Modawal, A. (2019, September 18). Smart vineyard - managing and monitoring made easy for wine producers . Retrieved from Softweb Solutions: https://www.softwebsolutions.com/resources/ vineyard-management-system.html 
Portincaso, M., \& de la Tour, A. (2019). The Dawn of the Deep Tech Ecosystem. Boston Consulting Group \& Hello Tomorrow.

Portincaso, M., de la Tour, A., \& Soussan, P. (2019, March 14). The BCG Henderson Institute. Retrieved from The Dawn of the Deep Tech Ecosystem : https://www.bcg.com/publications/2019/ dawn-deep-tech-ecosystem

Siegel, J., Krishnan, S., Subirana, B., Sarma, Sanjay, Merritt, J., . . . Arias, R. (2019). Realizing the Internet of Things: A Framework for Collective Action. World Economic Forum.

Soussan, P., \& de la Tour, A. (2017, April 3). What Deep-Tech Startups Want from Corporate Parnters. Retrieved from BCG Henderson Institute: https://www.bcg.com/publications/2017 /technology-digital-joint-ventures-alliances-what-deep-tech-startups-corporate-partners

Yahoo! (2020, September 10). Yahoo! Finance. Retrieved from Peloton Interactive, Inc. (PTON): https://finance.yahoo.com/quote/PTON/ 


\section{Biographies}

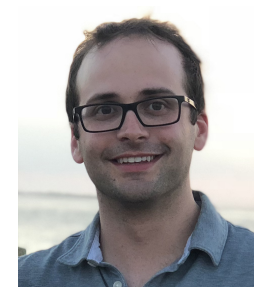

Joshua E. Siegel. Josh Siegel, Ph.D., is an Assistant Professor of Computer Science and Engineering at Michigan State University, where he runs the Deep Technology (DeepTech) Laboratory. He is also the creator of and lead instructor for the Massachusetts Institute of Technology's "Internet of Things" and "DeepTech" Bootcamps. His research focuses on cultivating Deep Technologies including pervasive sensing, secure and efficient network connectivity, universal diagnostics, the Internet of Things, and enhanced automated driving, and his graduate and undergraduate teaching encompasses self-driving, entrepreneurship, and the Internet of Things. Prior to joining Michigan State University, he was a Research Scientist at the Massachusetts Institute of Technology and the founder of CarKnow and DataDriven, two automotive technology companies. He holds Ph.D., S.M., and S.B. degrees in Mechanical Engineering from the Massachusetts Institute of Technology.

CRediT Statement: Conceptualization, Investigation, Project Administration, Supervision, Writing - Original Draft, Writing - Review \& Editing.

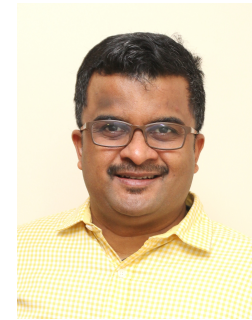

Sriram Krishnan. Dr. Sriram Krishnan is a Director and Principal Consultant at National Grid, a global energy company, and a Research Affiliate of the Mechanical Engineering Department at the Massachusetts Institute of Technology. Sriram has deep expertise in the solar energy industry where he served as a strategy consultant during the decade when the industry underwent exponential growth globally. As a consultant for McKinsey, Sriram has advised leading companies across industries on Product Development. In his academic background, Sriram is a peer reviewer for research relating to IoT, solar energy and manufacturing and advises doctoral researchers at MIT. He has published on the topics of IoT, manufacturing, machine design and CAD/CAM. He received his S.M. and Ph.D. degrees from MIT and a B.Tech degree from the Indian Institute of Technology, Madras, all in Mechanical engineering. Sriram is enthusiastic about building innovation ecosystems and actively mentors startups in the US and in India (in clean energy, technology platforms and retail e-commerce).

CRediT Statement: Conceptualization, Investigation, Writing - Original Draft, Writing - Review E Editing.

\section{ISSN 2183-0606}

http://www.open-jim.org

http://creativecommons.org/licenses/by/3.0 